\title{
ON THE INTEGRABILITY OF THE 5-DIMENSIONAL LORENZ SYSTEM FOR THE GRAVITY-WAVE ACTIVITY
}

\author{
JAUME LLIBRE ${ }^{1}$ AND CLÀUDIA VALLS ${ }^{2}$
}

AbStRACT. We consider the 5-dimensional Lorenz system

$$
\begin{aligned}
U^{\prime} & =-V W+b V Z, \\
V^{\prime} & =U W-b U Z, \\
W^{\prime} & =-U V, \\
X^{\prime} & =-Z, \\
Z^{\prime} & =b U V+X
\end{aligned}
$$

where $b \in \mathbb{R} \backslash\{0\}$ and the derivative is with respect to $T$. This system describes coupled Rosby waves and gravity waves. First we prove that the number of functionally independent global analytic first integrals of this differential system is two. This solves an open question in the paper On the analytic integrability of the 5-dimensional Lorenz system for the gravity-wave activity, Proc. Amer. Math. Soc. 142 (2014), 531-537, where it was proved that this number was two or three. Moreover, we characterize all the invariant algebraic surfaces of the system, and additionally we show that it has only two functionally independent Darboux first integrals.

\section{IntRoduction AND STATEMENT OF THE MAIN RESUlts}

E.N. Lorenz constructed in [9] a 5-dimensional 1-parameter differential system in $\mathbb{R}^{5}$ which describes coupled Rosby waves and gravity waves:

$$
\begin{aligned}
U^{\prime} & =-V W+b V Z, \\
V^{\prime} & =U W-b U Z, \\
W^{\prime} & =-U V, \\
X^{\prime} & =-Z, \\
Z^{\prime} & =b U V+X .
\end{aligned}
$$

He studied its slow manifolds and in this paper we are interested in studying its global analytic integrability, its algebraic invariant surfaces and its Darboux first integrals. More precisely, we want to know what is the maximal number of functionally independent either global analytic or Darboux first integrals that system (1) can exhibit?. This question has been considered for many other differential equations and other classes of first integrals not necessarily analytic or Darboux; see for instance $[6,7,10]$ and the references therein.

2010 Mathematics Subject Classification. Primary 37J35, 37K10.

Key words and phrases. Hamiltonian systems, weight-homogenous differential systems, polynomial integrability, rational integrability, Darboux polynomials, exponential factors, Darboux first integrals. 
Let $\Omega$ be an open subset of $\mathbb{R}^{5}$ invariant by the flow of the differential system (1). A first integral of the differential system (1) in $\Omega$ is a $C^{1}$-function $H$ satisfying

$$
\begin{aligned}
& (-V W+b V Z) \frac{\partial H}{\partial U}+(U W-b U Z) \frac{\partial H}{\partial V} \\
& \quad-U V \frac{\partial H}{\partial W}-Z \frac{\partial H}{\partial X}+(b U V+X) \frac{\partial H}{\partial Z} \equiv 0, \quad \text { in } \Omega .
\end{aligned}
$$

Let $H_{1}: \Omega_{1} \rightarrow \mathbb{R}$ and $H_{2}: \Omega_{2} \rightarrow \mathbb{R}$ be two first integrals of the 5-dimensional Lorenz system (1). They are functionally independent in $\Omega_{1} \cap \Omega_{2}$ if their gradients are linearly independent over a full Lebesgue measure subset of $\Omega_{1} \cap \Omega_{2}$.

From [9] we know that the 5-dimensional Lorenz system (1) has the polynomial first integrals

$$
H_{1}=U^{2}+V^{2} \text { and } H_{2}=V^{2}+W^{2}+X^{2}+Z^{2} .
$$

To study the existence of Darboux first integrals we will use the well-known Darboux theory of integrability. The Darboux theory of integrability in dimension 5 is based on the existence of invariant algebraic hypersurfaces (or Darboux polynomials). For more details see [2,3] and [5]. This theory is one of the best theories for studying the existence of first integrals for the polynomial differential systems.

A Darboux polynomial of system (1) is a polynomial $f \in \mathbb{C}[U, V, W, X, Z] \backslash \mathbb{C}$ such that

$$
\begin{aligned}
& (-V W+b V Z) \frac{\partial f}{\partial U}+(U W-b U Z) \frac{\partial f}{\partial V} \\
& -U V \frac{\partial f}{\partial W}-Z \frac{\partial f}{\partial X}+(b U V+X) \frac{\partial f}{\partial Z}=K f
\end{aligned}
$$

for some polynomial $K$ called the cofactor of $f$ and with degree at most one.

Note that $f=0$ is an invariant algebraic hypersurface for the flow of system (1). A polynomial first integral (a first integral which is a polynomial) is a Darboux polynomial with zero cofactor. We recall that if $f \notin \mathbb{R}[U, V, W, X, Z]$ is a Darboux polynomial then there exists another Darboux polynomial $\bar{f}$ (the conjugate of $f$ ) with cofactor $\bar{K}$ (the conjugate of $K$ ).

An exponential factor $F=F(U, V, W, X, Z)$ of system (1) is a function of the form $F=\exp \left(g_{0} / g_{1}\right) \notin \mathbb{C}$ with $g_{0}, g_{1} \in \mathbb{C}[U, V, W, X, Z]$ coprime satisfying that

$$
\begin{aligned}
& (-V W+b V Z) \frac{\partial F}{\partial U}+(U W-b U Z) \frac{\partial F}{\partial V} \\
& -U V \frac{\partial F}{\partial W}-Z \frac{\partial F}{\partial X}+(b U V+X) \frac{\partial F}{\partial Z}=L F
\end{aligned}
$$

for some polynomial $L=L(U, V, W, X, Z)$ called the cofactor of $F$ and with degree at most one. We recall that if $F \notin \mathbb{R}[U, V, W, X, Z 2]$ is an exponential factor then there exists another exponential factor $\bar{F}$ (the conjugate of $F$ ) with cofactor $\bar{L}$ (the conjugate of $L)$.

A Darboux first integral $G$ of system (1) is a first integral of the form

$$
G_{=} f_{1}^{\lambda_{1}} \cdots f_{p}^{\lambda_{p}} F_{1}^{\mu_{1}} \cdots F_{q}^{\mu_{q}}
$$

where $f_{1}, \ldots, f_{p}$ are Darboux polynomials and $F_{1}, \ldots, F_{q}$ are exponential factors and $\lambda_{j}, \mu_{k} \in \mathbb{C}$ for $j=1, \ldots, p$ and $k=1, \ldots, q$. Note that a Darboux first integral is always a real function due to the fact that if there are complex Darboux polynomials or complex exponential factors always also appear their conjugates. 
In [8] the authors studied system (1) from the view point of the analytic integrability. We recall that when $H$ is an analytic function we say that $H$ is an analytic first integral and when the domain of definition is $\mathbb{R}^{5}$ then $H$ is called a global analytic first integral. Additionally, they show that when $b=0$ the system is completely integrable with four functionally independent first integrals (not necessarily analytic), and that when $b \neq 0$ the number of functionally independent global analytic first integrals is either two or three.

In this paper first we prove that the differential system (1) has only two global analytic first integrals. After we characterize the Darboux first integrals of system (1). We recall that the class of Darboux first integrals and the class of global analytic first integrals have intersection but they are different classes. We also characterize the invariant algebraic surfaces and the so-called exponential factors.

Our main result is the following one.

Theorem 1. The following statements hold for the differential system (1) with $b \neq 0$.

(a) Any global analytic first integral must be a function in the variables $H_{1}$ and $\mathrm{H}_{2}$ given in (3).

(b) It has two irreducible Darboux polynomials $U+i V$ and $U-i V$ with non-zero cofactors $i(W-b Z)$ and $-i(W-b Z)$, respectively.

(c) It has only two functionally independent Darboux first integrals.

Theorem 1 (a) completes the characterization of the global analytic first integrals of system (1) which was unfinished in [8]. Theorem 1 (b) characterizes all its invariant algebraic hypersurfaces, and Theorem 1 (c) characterizes all the Darboux first integrals of system (1).

Theorem 1 (a) is proved in section 2. Theorem 1 (b) is proved in section 3 and Theorem 1 (c) is proved in section 4.

\section{Proof of Theorem 1(a)}

We prove the following proposition which is exactly the statement (a) of Theorem 1.

Proposition 2. Any global analytic first integral must be a function in the variables $H_{1}=U^{2}+V^{2}$ and $H_{2}=V^{2}+W^{2}+X^{2}+Y^{2}$.

Proof. Let $H$ be an analytic first integral of system (1) with $b \neq 0$. Then by definition we have that $H$ must satisfy (2). We expand $H$ in Taylor series

$$
H=\sum_{j=m}^{\infty} H_{j}(U, V, W, X, Z)
$$

where $m \geq 1$ is a positive integer, and $H_{j}$ for $j=m, m+1, \ldots$, are homogeneous polynomials of degree $j$. Comparing the homogeneous polynomials in (2) of the same degree, we get

$$
\begin{gathered}
-Z \frac{\partial H_{m}}{\partial X}+X \frac{\partial H_{m}}{\partial Z}=0 \\
-Z \frac{\partial H_{j+1}}{\partial X}+X \frac{\partial H_{j+1}}{\partial Z}= \\
\quad V(W-b Z) \frac{\partial H_{j}}{\partial U}-U(W-b Z) \frac{\partial H_{j}}{\partial V} \\
+U V \frac{\partial H_{j}}{\partial W}-b U V \frac{\partial H_{j}}{\partial Z},
\end{gathered}
$$


for $j=m, m+1, \ldots$. The characteristic equation associated with the linear partial differential equation (6) has the first integral $X^{2}+Z^{2}$, so by the method of characteristic curves for solving linear partial differential equations we get that the general solution of $(6)$ is

$$
H_{m}(U, V, W, X, Z)=\tilde{G}_{m}(A, U, V, W),
$$

where $\tilde{G}_{m}$ must be a polynomial function in its variables because $H_{m}$ is a homogeneous polynomial of degree $m$ in the variables $U, V, W, X, Z$.

For $j \geq m$ since $X^{2}+Z^{2}$ is a first integral of the characteristic equation associated with $-Z \bar{\partial} H_{j+1} / \partial X+X \partial H_{j+1} / \partial Z=0$ we make the change of variables

$$
A=X^{2}+Z^{2}, \quad Z=Z
$$

Then equation (7) becomes the ordinary differential equation

$$
\begin{aligned}
\sqrt{A-Z^{2}} \frac{d \tilde{H}_{j+1}}{d Z}= & V(W-b Z) \frac{\partial \tilde{H}_{j}}{\partial U}-U(W-b Z) \frac{\partial \tilde{H}_{j}}{\partial V} \\
& +U V \frac{\partial \tilde{H}_{j}}{\partial W}-2 b U V Z \frac{\partial \tilde{H}_{j}}{\partial A}
\end{aligned}
$$

where for $j \geq m, \tilde{H}_{j}$ is $H_{j}$ written in the variables $A, U, V, W, Z$ instead of the variables $U, V, W, X, Z$. Note that for $j=m$ we have $\tilde{H}_{m}=\tilde{G}_{m}(A, U, V, W)$ and

$$
\begin{aligned}
\frac{d \tilde{H}_{m+1}}{d Z}= & \left(V(W-b Z) \frac{\partial \tilde{G}_{m}}{\partial U}-U(W-b Z) \frac{\partial \tilde{G}_{m}}{\partial V}+U V \frac{\partial \tilde{G}_{m}}{\partial W}\right. \\
& \left.-2 b U V Z \frac{\partial \tilde{G}_{m}}{\partial A}\right) \frac{1}{\sqrt{A-Z^{2}}}=\frac{s_{1}+s_{2} Z}{\sqrt{A-Z^{2}}}
\end{aligned}
$$

where

$$
\begin{aligned}
& s_{1}=s_{1}(U, V, W, A)=V W \frac{\partial \tilde{G}_{m}}{\partial U}-U W \frac{\partial \tilde{G}_{m}}{\partial V}+U V \frac{\partial \tilde{G}_{m}}{\partial W} \\
& s_{2}=s_{2}(U, V, W, A)=-b V \frac{\partial \tilde{G}_{m}}{\partial U}+b U \frac{\partial \tilde{G}_{m}}{\partial V}-2 b U V \frac{\partial \tilde{G}_{m}}{\partial A} .
\end{aligned}
$$

Integrating this ordinary differential equation with respect to $Z$, we get

$$
\tilde{H}_{m+1}(A, U, V, W, Z)=s_{1} \arctan \frac{Z}{\sqrt{A-Z^{2}}}-s_{2} \sqrt{A-Z^{2}}+\tilde{G}_{m+1}(A, U, V, W),
$$

where $\tilde{G}_{m+1}$ is an integrating constant with respect to $Z$. Since $H_{m+1}=H_{m+1}(U, V, W, X, Z)$ is a homogeneous polynomial of degree $m+1$, we must have $s_{1}=0$, that is

$$
V W \frac{\partial \tilde{G}_{m}}{\partial U}-U W \frac{\partial \tilde{G}_{m}}{\partial V}+U V \frac{\partial \tilde{G}_{m}}{\partial W}=0
$$

The characteristic equations associated with this last partial differential equation (11) is $g(B, C)$, with $B=U^{2}+V^{2}, C=V^{2}+W^{2}$ and $g$ any continuous differentiable function. This forces that

$$
H_{m}(U, V, W, X, Z)=\tilde{G}_{m}(A, U, V, W)=R_{m}(A, B, C),
$$

with $R_{m}$ a homogeneous polynomial in the variables $A, B, C$. So $m$ must be even. Then $s_{2}$ becomes

$$
s_{2}=-2 b U V\left(\frac{\partial R_{m}}{\partial A}-\frac{\partial R_{m}}{\partial C}\right)=-2 b U V \tilde{S}_{m}, \quad \tilde{S}_{m}=\tilde{S}_{m}(A, B, C)=\frac{\partial R_{m}}{\partial A}-\frac{\partial R_{m}}{\partial C}
$$


Then

$$
\tilde{H}_{m+1}=2 b U V \sqrt{A-Z^{2}} \tilde{S}_{m}+\tilde{G}_{m+1}(A, U, V, W)
$$

Equation (9) with $j=m+1$ becomes

$$
\begin{aligned}
\sqrt{A-Z^{2}} \frac{d \tilde{H}_{m+2}}{d Z}= & V(W-b Z) \frac{\partial \tilde{H}_{m+1}}{\partial U}-U(W-b Z) \frac{\partial \tilde{H}_{m+1}}{\partial V}+U V \frac{\partial \tilde{H}_{m+1}}{\partial W} \\
& -2 b U V Z \frac{\partial \tilde{H}_{m+1}}{\partial A} \\
= & s_{3}+s_{4} Z+s_{5} \sqrt{A-Z^{2}}+s_{6} Z \sqrt{A-Z^{2}}-\frac{Z}{\sqrt{A-Z^{2}}} s_{7}
\end{aligned}
$$

where

$$
\begin{aligned}
s_{3}= & s_{3}(U, V, W, A)=V W \frac{\partial \tilde{G}_{m+1}}{\partial U}-U W \frac{\partial \tilde{G}_{m+1}}{\partial V}+U V \frac{\partial \tilde{G}_{m+1}}{\partial W}, \\
s_{4}= & s_{4}(U, V, W, A)=-b V \frac{\partial \tilde{G}_{m+1}}{\partial U}+b U \frac{\partial \tilde{G}_{m+1}}{\partial V}-2 b U V \frac{\partial \tilde{G}_{m+1}}{\partial A}, \\
s_{5}= & s_{5}(U, V, W, A)=V W\left(2 b V \tilde{S}_{m}+2 b U V \frac{\partial \tilde{S}_{m}}{\partial U}\right)-U W\left(2 b U \tilde{S}_{m}+2 b U V \frac{\partial \tilde{S}_{m}}{\partial V}\right) \\
& +2 b U^{2} V^{2} \frac{\partial \tilde{S}_{m}}{\partial W}, \\
s_{6}= & s_{6}(U, V, W, A)=-b V\left(2 b V \tilde{S}_{m}+2 b U V \frac{\partial \tilde{S}_{m}}{\partial U}\right)+b U\left(2 b U \tilde{S}_{m}+2 b U V \frac{\partial \tilde{S}_{m}}{\partial V}\right) \\
& -4 b^{2} U^{2} V^{2} \frac{\partial \tilde{S}_{m}}{\partial A}, \\
s_{7}= & s_{7}(U, V, W, A)=-4 b^{2} U^{2} V^{2} \tilde{S}_{m} .
\end{aligned}
$$

Solving it we get

$$
\begin{aligned}
\tilde{H}_{m+2}= & s_{3} \arctan \frac{Z}{\sqrt{A-Z^{2}}}-s_{4} \sqrt{A-Z^{2}}+s_{5} Z+\frac{s_{6}}{2} Z^{2} \\
& +\frac{s_{7}}{2} \log \left(Z^{2}-A\right)+\tilde{G}_{m+2}(A, U, V, W)
\end{aligned}
$$

where $\tilde{G}_{m+2}$ is an integrating constant with respect to $Z$. Since the polynomial $H_{m+2}=H_{m+2}(U, V, W, X, Z)$ is homogeneous of degree $m+2$, we must have $s_{3}=$ $s_{7}=0$. From $s_{3}=0$, as before for $s_{1}=0$, we get that $\tilde{G}_{m+1}$ must satisfy equation (11) with $\tilde{G}_{m+1}$ instead of $\tilde{G}_{m}$, and so $\tilde{G}_{m+1}=R_{m+1}(A, B, C)$, being $R_{m+1}$ a homogeneous polynomial of degree $m+1$. From $s_{7}=0$ we must have $\tilde{S}_{m}=0$. We write

$$
R_{m}(A, B, C)=\sum_{l=0}^{m} \sum_{k=0}^{m-l} a_{k, l} B^{l} A^{k} C^{m-l-k} .
$$


Hence

$$
\begin{aligned}
\tilde{S}_{m} & =\frac{\partial R_{m}}{\partial A}-\frac{\partial R_{m}}{\partial C} \\
& =\sum_{l=0}^{m} B^{l}\left(\sum_{k=0}^{m-l} k a_{k, l} A^{k-1} C^{m-l-k}-\sum_{k=0}^{m-l}(m-l-k) a_{k, l} A^{k} C^{m-l-k-1}\right) \\
& =\sum_{l=0}^{m} B^{l}\left(\sum_{k=0}^{m-l-1}\left((k+1) a_{k+1, l}-(m-l-k) a_{k, l}\right) A^{k} C^{m-l-k-1}\right)=0
\end{aligned}
$$

and so for each $l=0, \ldots, m$ we must have

$$
\sum_{k=0}^{m-l-1}\left((k+1) a_{k+1, l}-(m-l-k) a_{k, l}\right) A^{k} C^{m-l-k-1}=0 .
$$

That is

which yields

$$
a_{k, l}=a_{k-1, l} \frac{m-l-k+1}{k} \quad \text { for } \quad k=1, \ldots, m-l
$$

$$
a_{k, l}=a_{0, l}\left(\begin{array}{c}
m-l \\
k
\end{array}\right) .
$$

Hence, it follows from (15) that

$$
\begin{aligned}
R_{m}(A, B, C) & =\sum_{l=0}^{m} B^{l} \sum_{k=0}^{m-l} a_{0, l}\left(\begin{array}{c}
m-l \\
k
\end{array}\right) A^{k} C^{m-l-k} \\
& =\sum_{l=0}^{m} B^{l} a_{0, l}(A+C)^{m-l}=R_{m}(A+C, B) \\
& =R_{m}\left(V^{2}+W^{2}+X^{2}+Z^{2}, U^{2}+V^{2}\right)
\end{aligned}
$$

Moreover, from (12) using that $\tilde{S}_{m}=0$ and that $\tilde{G}_{m+1}=R_{m+1}(A, B, C)$ we get that

$$
\tilde{H}_{m+1}(A, U, V, W, Z)=R_{m+1}(A, B, C)
$$

Since $H_{m+1}$ is a homogeneous polynomial of odd degree, we must have

$$
\tilde{G}_{m+1} \equiv 0,
$$

because $\tilde{G}_{m+1}$ cannot be of odd degree by its expression. Then from (13) using that $\tilde{S}_{m}=\tilde{G}_{m+1}=0$, we obtain

$$
\tilde{H}_{m+2}=\tilde{G}_{m+2}(A, U, V, W), \quad \text { and } \quad H_{m+2}=G_{m+2}\left(X^{2}+Z^{2}, U, V, W\right),
$$

where $H_{m+2}$ is a homogeneous polynomial of degree $m+2$.

Proceeding by induction we can show that

$$
\begin{aligned}
\tilde{G}_{m+2 k}(A, U, V, W, Z) & =R_{m+2 k}(A+C, B) \\
& =R_{m+2 k}\left(V^{2}+W^{2}+X^{2}+Z^{2}, U^{2}+V^{2}\right), \\
\tilde{G}_{m+2 k-1}(A, U, V, W, Z) & =0,
\end{aligned}
$$

for $k=1, \ldots$, where $R_{m+2 k}$ are homogeneous functions in $A+C, B$. This proves the theorem. 


\section{Proof of Theorem $1(b)$}

To prove Theorem 1(b) we need to characterize the Darboux polynomials with non-zero cofactor.

We introduce the new variables $Y_{1}=U+i V$ and $Y_{2}=U-i V$ and rewrite system (1) in these new variables as

$$
\begin{aligned}
Y_{1}^{\prime} & =i Y_{1}(W-b Z), \\
Y_{2}^{\prime} & =-i Y_{2}(W-b Z), \\
W^{\prime} & =\frac{i}{4}\left(Y_{1}^{2}-Y_{2}^{2}\right), \\
X^{\prime} & =-Z, \\
Z^{\prime} & =-\frac{i b}{4}\left(Y_{1}^{2}-Y_{2}^{2}\right)+X .
\end{aligned}
$$

The proof of Theorem 1(b) can be reformulated as follows.

Theorem 3. The unique irreducible Darboux polynomials of system (16) with nonzero cofactor are $Y_{1}$ and $Y_{2}$ with cofactors $i(W-b Z)$ and $-i(W-b Z)$, respectively.

Let $f$ be a Darboux polynomial of system (16) with non-zero cofactor. Then $f$ satisfies

$$
\begin{aligned}
& i Y_{1}(W-b Z) \frac{\partial f}{\partial Y_{1}}-i Y_{2}(W-b Z) \frac{\partial f}{\partial Y_{2}}+\left(\frac{i}{4}\left(Y_{1}^{2}-Y_{2}^{2}\right)\right) \frac{\partial f}{\partial W} \\
& -Z \frac{\partial f}{\partial X}+\left(-\frac{i b}{4}\left(Y_{1}^{2}-Y_{2}^{2}\right)+X\right) \frac{\partial f}{\partial Z}=K f,
\end{aligned}
$$

where $K=\alpha_{0}+\alpha_{1} Y_{1}+\alpha_{2} Y_{2}+\alpha_{3} W+\alpha_{4} X+\alpha_{5} Z$ with $\alpha_{i} \in \mathbb{C}$ for $i=0, \ldots, 5$.

We write $K=\alpha_{0}+\alpha_{1} Y_{1}+\alpha_{2} Y_{2}+K_{1}$ where $K_{1}=\alpha_{3} W+\alpha_{4} X+\alpha_{5} Z$.

We separate the proof into the next two propositions.

Proposition 4. The unique irreducible Darboux polynomials of system (16) with cofactor $K_{1} \neq 0$ are $Y_{1}$ and $Y_{2}$ with cofactors $i(W-b Z)$ and $-i(W-b Z)$, respectively.

Proof. It is easy to check that the unique Darboux polynomials of system (16) of degree one with $K_{1} \neq 0$ are $Y_{1}$ and $Y_{2}$ with cofactors $i(W-b Z)$ and $-i(W-b Z)$, respectively. We will see that they are the unique irreducible ones.

Let $f=f\left(Y_{1}, Y_{2}, W, X, Z\right)$ be an irreducible Darboux polynomial of system (16) with degree $n \geq 2$ and with cofactor $K_{1} \neq 0$. We consider two different cases.

Case 1: $K_{1} \neq i \alpha(W-b Z)$ for any $\alpha>0$. We restrict system (16) to $Y_{2}=0$ that is we consider system

$$
\begin{aligned}
Y_{1}^{\prime} & =i Y_{1}(W-b Z), \\
W^{\prime} & =\frac{i}{4} Y_{1}^{2}, \\
X^{\prime} & =-Z, \\
Z^{\prime} & =-\frac{i b}{4} Y_{1}^{2}+X .
\end{aligned}
$$


Let $g=g\left(Y_{1}, W, X, Z\right)=f\left(Y_{1}, 0, W, X, Z\right)$ that is, the polynomial $f$ restricted to $Y_{2}=0$, so $g$ satisfies

$i Y_{1}(W-b Z) \frac{\partial g}{\partial Y_{1}}+\frac{i}{4} Y_{1}^{2} \frac{\partial g}{\partial W}-Z \frac{\partial g}{\partial X}+\left(-\frac{i b}{4} Y_{1}^{2}+X\right) \frac{\partial g}{\partial Z}=\left(\alpha_{0}+\alpha_{1} Y_{1}+K_{1}\right) g$.

We write $g$ as sum of its homogeneous parts as $g=\sum_{i=0}^{n} g_{i}$ where $g_{i}=g_{i}\left(Y_{1}, W, X, Z\right)$ is a homogeneous polynomial of degree $i$. Clearly, $g_{n}$ satisfies

$$
i Y_{1}(W-b Z) \frac{\partial g_{n}}{\partial Y_{1}}+\frac{i}{4} Y_{1}^{2} \frac{\partial g_{n}}{\partial W}-\frac{i b}{4} Y_{1}^{2} \frac{\partial g_{n}}{\partial Z}=\left(\alpha_{1} Y_{1}+K_{1}\right) g_{n} .
$$

We consider two subcases.

Subcase 1.1: $g_{n}$ is not divisible by $Y_{1}$. In this case if we restrict $g_{n}$ to $Y_{1}=0$ and denote it by $\bar{g}_{n}$ then $\bar{g}_{n} \neq 0$. Moreover $\bar{g}_{n}$ satisfies (19) restricted to $Y_{1}=0$. So $\bar{g}_{n}$ satisfies $0=K_{1} \bar{g}_{n}$, so $\bar{g}_{n}=0$ which is not possible.

Subcase 1.2: $g_{n}$ is divisible by $Y_{1}$. In this case we can write $g_{n}=Y_{1}^{\ell} h_{n}$ with $1 \leq \ell \leq n$ and $h_{n}$ is a homogeneous polynomial of degree $n-\ell$. If we restrict $h_{n}$ to $Y_{1}=0$ and denote it by $\bar{h}_{n}$ then $\bar{h}_{n} \neq 0$ and satisfies, after simplifying by $Y_{1}^{\ell}$, we get

(20) $i Y_{1}(W-b Z) \frac{\partial h_{n}}{\partial Y_{1}}+\frac{i}{4} Y_{1}^{2} \frac{\partial h_{n}}{\partial W}-\frac{i b}{4} Y_{1}^{2} \frac{\partial h_{n}}{\partial Z}=\left(\alpha_{1} Y_{1}+K_{1}-i \ell(W-b Z)\right) h_{n}$.

Now if we restrict $h_{n}$ to $Y_{1}=0$ and denote it by $\bar{h}_{n}$ then $\bar{h}_{n} \neq 0$. Moreover $\bar{h}_{n}$ satisfies (20) restricted to $Y_{1}=0$. So $\bar{h}_{n}$ satisfies

$$
0=\left(K_{1}-i \ell(W-b Z)\right) \bar{h}_{n},
$$

and by assumptions $K_{1}-i \ell(W-b Z) \neq 0$ which yields $\bar{g}_{n}=0$ which is not possible. Case 2: $K_{1}=i \alpha(W-b Z)$ for some $\alpha>0$. We restrict system (16) to $Y_{1}=0$ that is we consider system

$$
\begin{aligned}
Y_{2}^{\prime} & =-i Y_{2}(W-b Z), \\
W^{\prime} & =-\frac{i}{4} Y_{2}^{2}, \\
X^{\prime} & =-Z, \\
Z^{\prime} & =\frac{i b}{4} Y_{2}^{2}+X .
\end{aligned}
$$

Let $g=g\left(Y_{2}, W, X, Z\right)=f\left(0, Y_{2}, W, X, Z\right)$ that is, the polynomial $f$ restricted to $Y_{1}=0$, so $g$ satisfies

$$
-i Y_{2}(W-b Z) \frac{\partial g}{\partial Y_{2}}-\frac{i}{4} Y_{2}^{2} \frac{\partial g}{\partial W}-Z \frac{\partial g}{\partial X}+\left(\frac{i b}{4} Y_{2}^{2}+X\right) \frac{\partial g}{\partial Z}=\left(\alpha_{0}+\alpha_{2} Y_{2}+K_{1}\right) g .
$$

We write $g$ as sum of its homogeneous parts as $g=\sum_{i=0}^{n} g_{i}$ where $g_{i}=g_{i}\left(Y_{1}, W, X, Z\right)$ is a homogeneous polynomial of degree $i$. Clearly, $g_{n}$ satisfies

$$
-i Y_{2}(W-b Z) \frac{\partial g_{n}}{\partial Y_{2}}-\frac{i}{4} Y_{2}^{2} \frac{\partial g_{n}}{\partial W}+\frac{i b}{4} Y_{2}^{2} \frac{\partial g_{n}}{\partial Z}=\left(\alpha_{2} Y_{2}+K_{1}\right) g_{n} .
$$

We consider two subcases.

Subcase 2.1: $g_{n}$ is not divisible by $Y_{2}$. In this case if we restrict $g_{n}$ to $Y_{2}=0$ and denote it by $\bar{g}_{n}$ then $\bar{g}_{n} \neq 0$. Moreover $\bar{g}_{n}$ satisfies (22) restricted to $Y_{2}=0$. So $\bar{g}_{n}$ satisfies $0=K_{1} \bar{g}_{n}$ and so $\bar{g}_{n}=0$ which is not possible. 
Subcase 2.2: $g_{n}$ is divisible by $Y_{2}$. In this case we can write $g_{n}=Y_{2}^{\ell} h_{n}$ with $1 \leq \ell \leq n$ and $h_{n}$ is a homogeneous polynomial of degree $n-\ell$. If we restrict $h_{n}$ to $Y_{2}=0$ and denote it by $\bar{h}_{n}$ then $\bar{h}_{n} \neq 0$ and satisfies, after simplifying by $Y_{2}^{\ell}$, we get

$$
\begin{aligned}
& -i Y_{2}(W-b Z) \frac{\partial h_{n}}{\partial Y_{1}}-\frac{i}{4} Y_{2}^{2} \frac{\partial h_{n}}{\partial W}+\frac{i b}{4} Y_{2}^{2} \frac{\partial h_{n}}{\partial Z} \\
& =\left(\alpha_{2} Y_{2}+K_{1}+i \ell(W-b Z)\right) h_{n}=i(\ell+\alpha)(W-b Z) h_{n}
\end{aligned}
$$

Now if we restrict $h_{n}$ to $Y_{2}=0$ and denote it by $\bar{h}_{n}$ then $\bar{h}_{n} \neq 0$. Moreover $\bar{h}_{n}$ satisfies (23) restricted to $Y_{2}=0$. So $\bar{h}_{n}$ satisfies

$$
0=i(\ell+\alpha)(W-b Z) \bar{h}_{n},
$$

and by assumptions $\ell+\alpha>0$ which yields $\bar{h}_{n}=0$ which is not possible.

Now assume that $K_{1}=0$, so $K=\alpha_{0}+\alpha_{1} Y_{1}+\alpha_{2} Y_{2}$. We will prove the following proposition which together with Proposition 4 implies the proof of Theorem 1(b).

Proposition 5. System (16) has no irreducible Darboux polynomials with cofactor $K=\alpha_{0}+\alpha_{1} Y_{1}+\alpha_{2} Y_{2} \neq 0$.

Proof. Let $f$ be an irreducible Darboux polynomial with non-zero cofactor $K=$ $\alpha_{0}+\alpha_{1} Y_{1}+\alpha_{2} Y_{2} \neq 0$. Let $\tau: \mathbb{C}\left[Y_{1}, Y_{2}, W, X, Z\right] \rightarrow \mathbb{C}\left[Y_{1}, Y_{2}, W, X, Z\right]$ be the automorphism

$$
\tau\left(Y_{1}, Y_{2}, W, X, Z\right)=\left(-Y_{1},-Y_{2}, W, X, Z\right)
$$

and consider the polynomial $g=f \cdot \tau f$ that is invariant by $\tau$ with a cofactor of the form $K_{\tau}=2 \alpha_{0}$. We consider two different cases.

Case 1: $\alpha_{0}=0$. In this case since $K \neq 0$ we must have $\alpha_{1}^{2}+\alpha_{2}^{2} \neq 0$. Note that $g$ is a Darboux polynomial with zero cofactor, that is, it is a polynomial first integral. In view of Theorem $1(\mathrm{a})$ we must have $g=g\left(G_{1}, G_{2}\right)$, where

$$
\begin{aligned}
G_{1} & =H_{1}(U, V)=H_{1}\left(\frac{Y_{1}+Y_{2}}{2}, \frac{Y_{1}-Y_{2}}{2 i}\right)=Y_{1} Y_{2}, \\
G_{2} & =H_{2}(V, Y, X, Z)=H_{2}\left(\frac{Y_{1}-Y_{2}}{2 i}, Y, X, Z\right) \\
& =X^{2}+Z^{2}+W^{2}-\frac{1}{4}\left(Y_{1}^{2}+Y_{2}^{2}-2 Y_{1} Y_{2}\right) .
\end{aligned}
$$

Since $\tau\left(Y_{1}\right)=-Y_{1}, \tau\left(Y_{2}\right)=-Y_{2}$ and $\tau\left(G_{i}\right)=G_{i}$ for $i=1,2$, we must have $f=f\left(G_{1}, G_{2}\right)$. But then $f$ is a Darboux polynomial with zero cofactor, that is, $\alpha_{1}=\alpha_{2}=0$ which is not possible. So, this case is not possible.

Case 2: $\alpha_{0} \neq 0$. In this case, proceeding as in the proof of Proposition 4, let $\tilde{g}=$ $\tilde{g}\left(Y_{2}, W, X, Z\right)=g\left(0, Y_{2}, W, X, Z\right)$ that is, the polynomial $f$ restricted to $Y_{1}=0$, so $\tilde{g}$ satisfies

$$
-i Y_{2}(W-b Z) \frac{\partial \tilde{g}}{\partial Y_{1}}-\frac{i}{4} Y_{2}^{2} \frac{\partial \tilde{g}}{\partial W}-Z \frac{\partial \tilde{g}}{\partial X}+\left(\frac{i b}{4} Y_{2}^{2}+X\right) \frac{\partial \tilde{g}}{\partial Z}=2 \alpha_{0} \tilde{g}
$$

We write $\tilde{g}$ as sum of its homogeneous parts as $\tilde{g}=\sum_{i=0}^{n} \tilde{g}_{i}$ where $\tilde{g}_{i}=\tilde{g}_{i}\left(Y_{2}, W, X, Z\right)$ is a homogeneous polynomial of degree $i$. Clearly, $\tilde{g}_{n}$ satisfies

$$
-i Y_{2}(W-b Z) \frac{\partial \tilde{g}_{n}}{\partial Y_{2}}-\frac{i}{4} Y_{2}^{2} \frac{\partial \tilde{g}_{n}}{\partial W}+\frac{i b}{4} Y_{2}^{2} \frac{\partial \tilde{g}_{n}}{\partial Z}=0
$$


Solving this linear partial differential equation we get that $\tilde{g}_{n}=\tilde{g}_{n}(X, b W+$ $\left.Z,-Y_{2}^{2}+4 W\left(\left(1-b^{2}\right) W-2 b Z\right)\right)$, and since must be a polynomial of degree $n$ we obtain

$$
\tilde{g}_{n}=a X^{n-2 k-\ell}(b W+Z)^{\ell}\left(-Y_{2}^{2}+4 W\left(\left(1-b^{2}\right) W-2 b Z\right)\right)^{k} .
$$

Moreover $\tilde{g}_{n-1}$ satisfy

$$
\begin{aligned}
& -i Y_{2}(W-b Z) \frac{\partial \tilde{g}_{n-1}}{\partial Y_{2}}-\frac{i}{4} Y_{2}^{2} \frac{\partial \tilde{g}_{n-1}}{\partial W}+\frac{i b}{4} Y_{2}^{2} \frac{\partial \tilde{g}_{n-1}}{\partial Z} \\
& =a X^{-2 k-\ell+n-1}(b W+Z)^{\ell-1}\left(-4 b^{2} W^{2}+4 W^{2}-8 b Z W-Y_{2}^{2}\right)^{k-1}\left(\left(\ell X^{2}-\right.\right. \\
& \left.2 b W \alpha_{0} X-2 Z \alpha_{0} X+2 k Z^{2}+\ell Z^{2}-n Z^{2}+2 b k W Z+b \ell W Z-b n W Z\right) Y_{2}^{2} \\
& +4 W\left(2 k W^{2} Z b^{3}+\ell W^{2} Z b^{3}-n W^{2} Z b^{3}-2 W^{2} X \alpha_{0} b^{3}+2 k W X^{2} b^{2}+\right. \\
& \ell W X^{2} b^{2}+6 k W Z^{2} b^{2}+3 \ell W Z^{2} b^{2}-3 n W Z^{2} b^{2}-6 W X Z \alpha_{0} b^{2}+4 k Z^{3} b+ \\
& 2 \ell Z^{3} b-2 n Z^{3} b-2 k W^{2} Z b-\ell W^{2} Z b+n W^{2} Z b+2 k X^{2} Z b+2 \ell X^{2} Z b- \\
& \left.\left.4 X Z^{2} \alpha_{0} b+2 W^{2} X \alpha_{0} b-\ell W X^{2}-2 k W Z^{2}-\ell W Z^{2}+n W Z^{2}+2 W X Z \alpha_{0}\right)\right) .
\end{aligned}
$$

Restricting it to $Y_{2}=0$ and using that $\alpha_{0} \neq 0$ we obtain that $\tilde{g}_{n}=0$ and so $g$ is divisible by $Y_{1}$. Proceeding in the same way we get that $g$ must be divisible by $Y_{2}$ and so $g=Y_{1} Y_{2} h$. Therefore, $h$ is a homogeneous polynomial of degree $n-2$. Then $h$ also satisfies the same as $g$ because $Y_{1} Y_{2}$ is a first integral. Proceeding as we did, we get that $h$ is divisible by $Y_{1} Y_{2}$. Proceeding inductively, we get that $n$ is even and $g=\left(Y_{1} Y_{2}\right)^{n / 2} a_{0}$. So $g$ is a first integral with zero cofactor, a contradiction. This concludes the proof.

\section{Proof of Theorem $1(c)$}

Note that it follows from Theorem 1(a) that the unique Darboux polynomials of system (1) with zero cofactor (that is, the polynomial first integrals) of system (1) are polynomials in the variables $H_{1}$ and $H_{2}$.

We introduce several auxiliary results. The first one was proved in [4].

Lemma 6. Let $f$ be a polynomial and $f=\prod_{j=1}^{s} f_{j}^{\alpha_{j}}$ its decomposition into irreducible factors in $\mathbb{C}[U, V, W, X, Z]$. Then $f$ is a Darboux polynomial if and only if all the $f_{j}$ are Darboux polynomials. Moreover, if $K$ and $K_{j}$ are the cofactors of $f$ and $f_{j}$, then $K=\sum_{j=1}^{s} \alpha_{j} K_{j}$.

In view of Theorem 1(a) if $f$ is a Darboux polynomial (or $f=0$ is an invariant algebraic surface) then $f=Y_{1}^{n_{1}} Y_{2}^{n_{2}} G_{2}^{n_{3}}$ where $n_{1}, n_{2}, n_{3} \in \mathbb{N} \cup\{0\}$ (see (25)). The second result that we will need is proved in [1].

Proposition 7. The following statements hold.

(a) If $E=\exp \left(g_{0} / g\right)$ is an exponential factor for the polynomial system (16) and $g$ is not a constant polynomial, then $g=0$ is an invariant algebraic hypersurface. 
(b) Eventually $e^{g_{0}}$ can be an exponential factor, coming from the multiplicity of the infinite invariant hyperplane.

In view of the above explanation and Proposition 7 if $F=\exp (h / g)$ is an exponential factor of system (16) then it must be of the form $F=\exp \left(h /\left(Y_{1}^{n_{1}} Y_{2}^{n_{2}} G_{2}^{n_{3}}\right)\right)$ with $h \in \mathbb{C}\left[Y_{1}, Y_{2}, W, X, Z\right]$ and $n_{1}, n_{2}, n_{3} \in \mathbb{N} \cup\{0\}$, with $h$ coprime with $Y_{1}$ if $n_{1}>0 ; h$ coprime with $Y_{2}$ if $n_{2}>0$ and $h$ coprime with $G_{2}$ if $n_{3}>0$. Moreover, since the cofactor must have at most degree one we can write it as $L=\beta_{0}+\beta_{1} Y_{1}+\beta_{2} Y_{2}+\beta_{3} W+\beta_{4} X+\beta_{5} Z$. So, $h$ must satisfy,

$$
\begin{aligned}
& i Y_{1}(W-b Z) \frac{\partial h}{\partial Y_{1}}-i Y_{2}(W-b Z) \frac{\partial h}{\partial Y_{2}}+\frac{i}{4}\left(Y_{1}^{2}-Y_{2}^{2}\right) \frac{\partial h}{\partial W}-Z \frac{\partial h}{\partial X} \\
& \quad+\left(-\frac{i b}{4}\left(Y_{1}^{2}-Y_{2}^{2}\right)+X\right) \frac{\partial h}{\partial Z}-i\left(n_{1}-n_{2}\right)(W-b Z) h \\
& =Y_{1}^{n_{1}} Y_{2}^{n_{2}} G_{2}^{n_{3}}\left(\beta_{0}+\beta_{1} Y_{1}+\beta_{2} Y_{2}+\beta_{3} W+\beta_{4} X+\beta_{5} Z\right) .
\end{aligned}
$$

We will prove that $n_{1}=n_{2}$. Indeed, we consider two cases.

Case 1: $n_{1}>n_{2}$. In this case, evaluating (26) on $Y_{1}=0$, and denoting by $\bar{h}$ the restriction of $h$ to $Y_{1}=0$, that is $\bar{h}=\bar{h}\left(Y_{2}, W, X, Z\right)=h\left(0, Y_{2}, W, X, Z\right)$ we have

$$
\begin{aligned}
& -i Y_{2}(W-b Z) \frac{\partial \bar{h}}{\partial Y_{2}}-\frac{i}{4} Y_{2}^{2} \frac{\partial \bar{h}}{\partial W}-Z \frac{\partial \bar{h}}{\partial X}+\left(\frac{i b}{4} Y_{2}^{2}+X\right) \frac{\partial \bar{h}}{\partial Z} \\
& =i\left(n_{1}-n_{2}\right)(W-b Z) \bar{h} .
\end{aligned}
$$

So $\bar{h}$ is either zero or a Darboux polynomial of system (21) with cofactor $i\left(n_{1}-\right.$ $\left.n_{2}\right)(W-b Z)$. The first case is not possible because $h$ is coprime with $Y_{1}$. The other case is also not possible because the cofactor of $\bar{h}$ is of the form $i \alpha(W-b Z)$ for some $\alpha>0$, and so it follows from the proof of Case 2 in Proposition 4 that $\bar{h}=0$ which is not possible.

Case 2: $n_{1}<n_{2}$. In this case, evaluating (26) on $Y_{2}=0$, and denoting by $\bar{h}$ the restriction of $h$ to $Y_{2}=0$, we obtain

$$
\begin{aligned}
& i Y_{1}(W-b Z) \frac{\partial \bar{h}}{\partial Y_{2}}+\frac{i}{4} Y_{1}^{2} \frac{\partial \bar{h}}{\partial W}-Z \frac{\partial \bar{h}}{\partial X}+\left(-\frac{i b}{4} Y_{1}^{2}+X\right) \frac{\partial \bar{h}}{\partial Z} \\
& =i\left(n_{1}-n_{2}\right)(W-b Z) \bar{h} .
\end{aligned}
$$

So $\bar{h}$ is either zero or a Darboux polynomial of system (18) with cofactor $i\left(n_{1}-\right.$ $\left.n_{2}\right)(W-b Z)$. The first case is not possible because $h$ is coprime with $Y_{2}$. The other case is also not possible because the cofactor of $\bar{h}$ is of the form $-i \alpha(W-b Z)$ for some $\alpha>0$, and so it follows from the proof of Case 1 in Proposition 4 that $\bar{h}=0$ which is not possible.

Hence $n_{1}=n_{2}$ and $F=\exp \left(h /\left(G_{1}^{n_{1}} G_{2}^{n_{3}}\right)\right)$ and satisfies

$$
\begin{aligned}
& i Y_{1}(W-b Z) \frac{\partial h}{\partial Y_{1}}-i Y_{2}(W-b Z) \frac{\partial h}{\partial Y_{2}}+\frac{i}{4}\left(Y_{1}^{2}-Y_{2}^{2}\right) \frac{\partial h}{\partial W}-Z \frac{\partial h}{\partial X} \\
& \quad+\left(-\frac{i b}{4}\left(Y_{1}^{2}-Y_{2}^{2}\right)+X\right) \frac{\partial h}{\partial Z} \\
& =G_{1}^{n_{1}} G_{2}^{n_{3}}\left(\beta_{0}+\beta_{1} Y_{1}+\beta_{2} Y_{2}+\beta_{3} W+\beta_{4} X+\beta_{5} Z\right) .
\end{aligned}
$$

Now we need the following result whose proof can be found in [4]. 
Theorem 8. Suppose that system (16) admits p Darboux polynomials $f_{i}$ with cofactors $k_{i}$ and $q$ exponential factors $F_{j}$ with cofactors $\ell_{j}$. Then there exists $\lambda_{j}, \mu_{j} \in \mathbb{C}$ not all zero such that

$$
\sum_{i=1}^{q} \lambda_{k} k_{i}+\sum_{i=1}^{q} \mu_{i} \ell_{i}=0
$$

if and only if the function $G$ given in (5) (called of Darboux type) is a first integral of system (16).

In view of Theorem 8 if $G$ is a Darboux first integral it must be of the form

$$
G=Y_{1}^{\lambda_{1}} Y_{2}^{\lambda_{2}} G_{2}^{\lambda_{3}} \exp \left(\mu_{1} h /\left(G_{1}^{n_{1}} G_{2}^{n_{3}}\right)\right)
$$

whose cofactor $K_{G}$ is

$$
K_{G}=i\left(\lambda_{1}-\lambda_{2}\right)(W-b Z)+\mu_{1}\left(\beta_{0}+\beta_{1} Y_{1}+\beta_{2} Y_{2}+\beta_{3} W+\beta_{4} X+\beta_{5} Z\right) .
$$

Since $G$ is a first integral we must have $K_{G}=0$. This yields that either $\mu_{1}=0$ and $\lambda_{1}=\lambda_{2}$ or $\mu_{1} \neq 0$ and $\beta_{0}=\beta_{1}=\beta_{2}=\beta_{4}=0, \beta_{5}=-b \beta_{3}$ and $\lambda_{1}-\lambda_{2}=-i \beta_{3} \mu_{1}$. In the first case $G=G_{1}^{\lambda_{1}} G_{2}^{\lambda_{2}}$ and so $G$ is a Darboux first integral in the variables $G_{1}$ and $G_{2}$.

In the second case we have $F=\exp \left(h /\left(G_{1}^{n_{1}} G_{2}^{n_{2}}\right)\right)$ with cofactor $L=\beta_{3}(W-b Z)$. Imposing this in (27) we get that $h$ must satisfy

$$
\begin{aligned}
& i Y_{1}(W-b Z) \frac{\partial h}{\partial Y_{1}}-i Y_{2}(W-b Z) \frac{\partial h}{\partial Y_{2}}+\frac{i}{4}\left(Y_{1}^{2}-Y_{2}^{2}\right) \frac{\partial h}{\partial W}-Z \frac{\partial h}{\partial X} \\
& \quad+\left(-\frac{i b}{4}\left(Y_{1}^{2}-Y_{2}^{2}\right)+X\right) \frac{\partial h}{\partial Z}=\beta_{3}(W-b Z) G_{1}^{n_{1}} G_{2}^{n_{2}} .
\end{aligned}
$$

We will show that $\beta_{3}=0$. We consider different cases. If $\operatorname{deg} h \leq 2 n_{1}+2 n_{3}$ it follows from (28) that $\beta_{3}=0$. So, $\operatorname{deg} h \geq 2 n_{1}+2 n_{3}+1$. Moreover, if $\operatorname{deg} h=$ $2 n_{1}+2 n_{3}+1$ then it follows from (28) that

$$
-Z \frac{\partial h}{\partial X}+X \frac{\partial h}{\partial Z}=\beta_{3} G_{1}^{n_{1}} G_{3}^{n_{3}}(W-b Z)
$$

With the change of variables in (8) and using the notation of Proposition 7 we have that the above equation is equivalent to

$$
\sqrt{A-Z^{2}} \frac{d \tilde{h}}{d Z}=\beta_{3}\left(U^{2}+V^{2}\right)^{n_{1}}\left(V^{2}+W^{2}+A^{2}\right)^{n_{3}}(W-b Z) .
$$

Proceeding again as in (10) with

$$
\begin{aligned}
& s_{1}=\beta_{3}\left(U^{2}+V^{2}\right)^{n_{1}}\left(V^{2}+W^{2}+A^{2}\right)^{n_{3}} W, \\
& s_{2}=-b \beta_{3}\left(U^{2}+V^{2}\right)^{n_{1}}\left(V^{2}+W^{2}+A^{2}\right)^{n_{3}},
\end{aligned}
$$

we conclude that $s_{1}=0$ and so $\beta_{3}=0$. Hence, we can assume that $\operatorname{deg} h>$ $2 n_{1}+2 n_{3}+1$. We write $\operatorname{deg} h=2 n_{1}+2 n_{3}+1+k$ for some $k>0$. Proceeding as in the proof of Proposition 7 we get that if we expand $h$ is its homogeneous parts as $h=\sum_{j=m}^{2 n_{1}+2 n_{3}+1+k} h_{j}$, then $m$ is even and $h_{2 j}=h_{2 j}(A+C, B), h_{2 j+1}=0$ for $j=m / 2, \ldots, n_{1}+n_{3}-1$. Then $h_{2 n_{1}+2 n_{3}}$ satisfies (6) and proceeding as in proof of 
Proposition 7 we get that $h_{2 n_{1}+2 n_{3}}=\tilde{G}_{2 n_{1}+2 n_{3}}(A, U, V, W)$ and $h_{2 n_{1}+2 n_{3}+1}$ satisfies

$$
\begin{aligned}
\frac{d \tilde{h}_{2 n_{1}+2 n_{3}+1}}{d Z}= & \left(V(W-b Z) \frac{\partial \tilde{G}_{2 n_{1}+2 n_{3}}}{\partial U}-U(W-b Z) \frac{\partial \tilde{G}_{2 n_{1}+2 n_{3}}}{\partial V}+U V \frac{\partial \tilde{G}_{2 n_{1}+2 n_{3}}}{\partial W}\right. \\
& \left.-2 b U V Z \frac{\partial \tilde{G}_{2 n_{1}+2 n_{3}}}{\partial A}+\beta_{3} G_{1}^{n_{1}} G_{3}^{n_{3}}(W-b Z)\right) \frac{1}{\sqrt{A-Z^{2}}}=\frac{s_{1}+s_{2} Z}{\sqrt{A-Z^{2}}},
\end{aligned}
$$

which is (10) with

$$
\begin{aligned}
s_{1}= & s_{1}(U, V, W, A)=V W \frac{\partial \tilde{G}_{2 n_{1}+2 n_{3}}}{\partial U}-U W \frac{\partial \tilde{G}_{2 n_{1}+2 n_{3}}}{\partial V}+U V \frac{\partial \tilde{G}_{2 n_{1}+2 n_{3}}}{\partial W} \\
& +\beta_{3}\left(U^{2}+V^{2}\right)^{n_{1}}\left(V^{2}+W^{2}+A^{2}\right)^{n_{3}} W, \\
s_{2}= & s_{2}(U, V, W, A)=-b V \frac{\partial \tilde{G}_{2 n_{1}+2 n_{3}}}{\partial U}+b U \frac{\partial \tilde{G}_{2 n_{1}+2 n_{3}}}{\partial V}-2 b U V \frac{\partial \tilde{G}_{2 n_{1}+2 n_{3}}}{\partial A} \\
& -b \beta_{3}\left(U^{2}+V^{2}\right)^{n_{1}}\left(V^{2}+W^{2}+A^{2}\right)^{n_{3}} .
\end{aligned}
$$

Proceeding again as in the proof of Proposition 7 we get that $s_{1}=0$ that is

$$
\begin{gathered}
V W \frac{\partial \tilde{G}_{2 n_{1}+2 n_{3}}}{\partial U}-U W \frac{\partial \tilde{G}_{2 n_{1}+2 n_{3}}}{\partial V}+U V \frac{\partial \tilde{G}_{2 n_{1}+2 n_{3}}}{\partial W} \\
+\beta_{3}\left(U^{2}+V^{2}\right)^{n_{1}}\left(V^{2}+W^{2}+A^{2}\right)^{n_{3}} W=0 .
\end{gathered}
$$

Introducing the change of variables $B=U^{2}+V^{2}, C=V^{2}+W^{2}$ with inverse change $U=\sqrt{B-V^{2}}, W=\sqrt{C-V^{2}}$ we rewrite $s_{1}=0$ as

$$
\sqrt{B-V^{2}} \sqrt{C-V^{2}} \frac{d \hat{G}_{2 n_{1}+2 n_{3}}}{d V}=\beta_{3} \sqrt{C-V^{2}} B^{n_{1}}\left(C^{2}+A^{2}\right)^{n_{3}},
$$

where $\hat{G}_{2 n_{1}+2 n_{3}}=\hat{G}_{2 n_{1}+2 n_{3}}(A, B, C)=\tilde{G}_{2 n_{1}+2 n_{3}}(A, U, V, W)$. Solving this differential equation we obtain

$$
\hat{G}_{2 n_{1}+2 n_{3}}=\beta_{3} B^{n_{1}}\left(C^{2}+A^{2}\right)^{n_{3}} \arctan \frac{V}{\sqrt{B-V^{2}}} .
$$

Since $G_{2 n_{1}+2 n_{3}}$ must be a polynomial we must have $\beta_{3}=0$.

We have proved that $\beta_{3}=0$, and so $\beta_{i}=0$ for $i=0, \ldots, 5$. This implies that $L=0$ and $h$ must be a polynomial first integral. In view of Theorem 1(a) we have $h=h\left(G_{1}, G_{2}\right)$. Moreover $\lambda_{1}=\lambda_{2}$ because $\beta_{3}=0$ and so $G=G_{1}^{\lambda_{1}} G_{2}^{\lambda_{3}} \exp \left(h\left(G_{1}, G_{2}\right) /\left(G_{1}^{n_{1}} G_{2}^{n_{3}}\right)\right.$ which clearly implies that $G$ is a Darboux first integral in the variables $G_{1}$ and $G_{2}$. This concludes the proof of Theorem 1(c).

\section{ACKNOWLEDGEMENTS}

The first author is partially supported by MINECO/FEDER grant number MTM2013-40998-P, an AGAUR grant number 2014SGR-568 and the grants FP7PEOPLE-2012-IRSES 318999 and 316338. The second author is partially supported by FCT/Portugal through UID/MAT/04459/2013.

\section{REFERENCES}

[1] C. Christopher, J. Llibre and J.V. Pereira, Multiplicity of invariant algebraic curves in polynomial vector fields, Pacific J. Math. 229 (2007), 63-117.

[2] G. Darboux, Mémoire sur les équations différentielles du premier ordre et du premier degreé (Mélanges), Bull. Sci. Math. 2éme série 2 (1878), 60-96, 123-144, 151-200. 
[3] G. Darboux, De l'emploi des solutions particulières algébriques dans l'intégration des systèmes d'équations différentielles algébriques, C. R. Math. Acad. Sci. Paris 86 (1878), $1012-1014$.

[4] F. Dumortier, J. Llibre and J.C. ARTÉs, Qualitative theory of planar differential systems, Universitext, Springer-Verlag, Berlin, 2006.

[5] J. Llibre And X. Zhang, Polynomial first integrals for quasi-homogeneous polynomial differential systems, Nonlinearity 15 (2002), 1269-1280.

[6] A.J. Maciejewski, M. Przybylska, T. Stachowiak and M. Szydlowski, Global integrability of cosmological scalar fields, J. Phys. A 41 (2008), 26pp. 465101.

[7] I.A. García, M. Grau and J. Llibre, First integrals and Darboux polynomials of natural polynomial Hamiltonian systems, Phys. Lett. A 374 (2010), 4746-4748.

[8] J. Llibre, R. Saghin and X. Zhang, On the analytic integrability of the 5-dimensional Lorenz system for the gravity-wave activity, Proc. Amer. Math. Soc. 142 (2014), 531-537.

[9] E.N. Lorenz, On the existence of a slow manifold, J. Atmospheric Sci. 43 (1986), 1547-1557.

[10] S.I. Popov and J..M. Strelcyn, On rational integrability of Euler equation on Lie algebra so $(4, \mathbb{C})$, revisited, Phys. Lett. A 373 (2009), 2445-2453.

1 Departament de Matemàtiques, Universitat Autònoma de Barcelona, 08193 Bellaterra, Barcelona, Catalonia, Spain

E-mail address: jllibre@mat.uab.cat

2 Departamento de Matemática, Instituto Superior Técnico, Universidade Técnica de Lisboa, Av. Rovisco Pais 1049-001, Lisboa, Portugal

E-mail address: cvalls@math.ist.utl.pt 